\title{
Simplex-Coded BOTDA Sensor Over 120-km SMF With 1-m Spatial Resolution Assisted by Optimized Bidirectional Raman Amplification
}

\author{
Marcelo A. Soto, Mohammad Taki, Gabriele Bolognini, Member, IEEE, \\ and Fabrizio Di Pasquale, Member, IEEE
}

\begin{abstract}
Bidirectional low-noise Raman amplification and simplex coding based on the return-to-zero modulation format are optimized through numerical simulations for long-range Brillouin optical time-domain analysis sensing. Experimental results are reported on sensing capabilities along $120-\mathrm{km}$ distance with 1-m spatial resolution, and worst-case temperature and strain resolution values of $1.3{ }^{\circ} \mathrm{C}$ and $26 \mu \varepsilon$, respectively.
\end{abstract}

Index Terms-Brillouin scattering, fiber optics sensors, Raman amplification, strain and temperature measurements.

\section{INTRODUCTION}

$\mathbf{T}$ HE ABILITY of Brillouin optical time-domain analysis (BOTDA) [1] to measure distributed temperature and strain with meter spatial resolution along tens of kilometers of optical fiber has taken the attention of many industrial sectors.

In fact, significant research efforts involve overcoming the main factors limiting the performance of BOTDA sensors, in particular those related to the well-known trade-off between signal-to-noise ratio (SNR) and sensing range. Thus, while the maximum power levels allowed in the sensor are limited by pump depletion [1], [2] and nonlinear effects, several methods have been recently proposed to overcome the above mentioned trade-off and to further enhance the performance of BOTDA sensors. In particular, optical pulse coding (OPC) techniques [3], [4] and distributed Raman amplification (DRA) [5], [6] have allowed for sensing ranges beyond $100 \mathrm{~km}$, but with a spatial resolution limited to a few meters. Pulse coding has also been combined with DRA [7], where, however, the use of Raman pumps with high relative intensity noise (RIN) and non-optimized power levels led to a noisy system with a range limited to $75 \mathrm{~km}$ and a spatial resolution of $2.5 \mathrm{~m}$.

In this letter, in order to enhance the performance of longrange BOTDA sensors, we combine the use of an optimized bidirectional DRA and Simplex OPC. The power levels of the Raman pumps, Brillouin pump and probe signal are optimized

Manuscript received May 29, 2012; revised July 26, 2012; accepted August 1, 2012. Date of publication August 7, 2012; date of current version September 26, 2012.

M. A. Soto, M. Taki, and F. Di Pasquale are with the Scuola Superiore Sant'Anna, TeCIP Institute, Pisa 56124, Italy (e-mail: m.soto@sssup.it; m.taki@sssup.it; f.dipasquale@sssup.it).

G. Bolognini is with the Consiglio Nazionale delle Ricerche, IMM Institute, Bologna 40129, Italy (e-mail: bolognini@bo.imm.cnr.it).

Color versions of one or more of the figures in this letter are available online at http://ieeexplore.ieee.org.

Digital Object Identifier 10.1109/LPT.2012.2212183 through numerical simulations. With the use of optimized low-RIN Raman pumps and optimized return-to-zero (RZ) OPC, we demonstrate a large SNR enhancement in long-range BOTDA sensors, reporting, for first time to our knowledge, distributed measurements over $120 \mathrm{~km}$ with $1.3{ }^{\circ} \mathrm{C}(26 \mu \varepsilon)$ temperature (strain) resolution and $1 \mathrm{~m}$ spatial resolution.

\section{Optimization OF RAMAN-Assisted CODED BOTDA}

In BOTDA sensors, a pulsed pump amplifies a counterpropagating continuous-wave $(\mathrm{CW})$ probe signal by stimulated Brillouin scattering (SBS) [1]. The strain- and temperaturedependent Brillouin gain spectrum (BGS) is measured by sweeping the probe-pump frequency difference, so that the Brillouin frequency shift (BFS) parameter can then be estimated along the fiber [1]. Methods such as OPC or DRA have been proposed to enhance the performance of BOTDA sensors [4]-[6], reaching more than $100 \mathrm{~km}$ distance with spatial resolution values of 2-3 m. Improving the spatial resolution down to $1 \mathrm{~m}$ along such a long range is considered particularly challenging since the acoustic-wave decay time $(\sim 10 \mathrm{~ns})$ induces a considerable BGS broadening when approaching $1 \mathrm{~m}$ resolution, leading also to a reduced Brillouin gain. Both issues strongly degrade the accuracy of the estimated BFS, resulting in a significantly worsened measurand resolution.

In this letter we aim at the demonstration of a $120 \mathrm{~km}$-long BOTDA sensor with $1 \mathrm{~m}$ spatial resolution using low-RIN Raman pumping and an optimized RZ-OPC. Simulations are carried out to optimize the optical SNR (OSNR) of the probe reaching the receiver, avoiding at the same time nonlinear effects and pump depletion [2]. Considering the fast response time of stimulated Raman scattering (SRS) and the use of 10 ns Simplex-coded pulses, a quasi-CW SRS model can be assumed to be valid. This can also be justified by the relatively long Brillouin pump pulse duration in comparison with the chromatic-dispersion-induced walk-off between Raman pumps and Brillouin pump or probe. Moreover, the equations describing bidirectional DRA of Brillouin pumpprobe can be separately solved with respect to those describing the SBS amplification in case of coded pulses. Actually, counter-propagating DRA results in negligible Raman pump depletion due to the low power levels of the Brillouin signals (pump and probe waves), and to amplification of Brillouin signals independently on their input power. On the other side, also co-propagating DRA of Brillouin pump-probe results 
to be independent of the specific coding scheme, codeword or bit length. In fact, considering the effective length of Raman amplification in our fiber $L_{\text {eff }} \sim 14.4 \mathrm{~km}$ [8], then the maximum walk-off $\Delta T$ between co-Raman pump and Brillouin pump-probe is equal to $\Delta \mathrm{T}=D_{0} \cdot L_{e f f} \cdot \Delta \lambda$ $\sim 16$ ns [8], i.e. the walk-off is limited to a fraction of a single bit slot ( $80 \mathrm{~ns}$ ) when using RZ format as explained below, and hence, DRA is independent of the code scheme or codeword. Under these assumptions, the following system of differential equations allows one to obtain the optimized power levels for forward and backward Raman pumps, for the Brillouin pump and probe signal [6]:

$$
\begin{aligned}
\frac{d}{d z} P_{i}^{ \pm}(z)= & \mp \alpha_{i} P_{i}^{ \pm}(z) \pm \gamma_{i} P_{i}^{ \pm}(z) \pm \sum_{j} C_{i j} P_{i}^{ \pm}(z) \\
& \times\left[P_{j}^{+}(z)+P_{j}^{-}(z)\right] \\
& \pm 2 h v_{i}^{ \pm} \Delta v \sum_{j} C_{i j}\left[P_{j}^{+}(z)+P_{j}^{-}(z)\right]
\end{aligned}
$$

where $i$ and $j$ subscripts refer to the signals at $\lambda_{i}$ and $\lambda_{j}$, respectively, $P_{i}^{+}(z)$ is the power of forward propagating signals (i.e. the Brillouin pump, forward Raman pump and the respective ASE light), $P_{i}^{-}(z)$ represents the power of backward propagating waves (i.e. the probe signal, backward Raman pump and the backward-propagating ASE noise). $\alpha_{i}$ is the fiber attenuation, $\gamma_{i}$ is the Rayleigh scattering coefficient, $f_{i}$ is the optical frequency corresponding to $\lambda_{i}, h$ is the Planck constant, $\Delta v$ is the bandwidth for ASE light calculation, and $C_{i j}$ is the Raman gain/depletion term between $\lambda_{i}$ and $\lambda_{j}$.

The equation system has been solved numerically for a wide range of input power levels, following a multi-step iteration scheme along forward and backward directions [6]. Numerical optimization of forward-propagating signals [6] aimed at maximizing the Brillouin pump power at the fiber end, while keeping its maximum level along the fiber below the threshold of nonlinear effects. The maximum input probe power with coding depends on peak pump power, determining the overall Brillouin gain and the amount of pump depletion. Hence the optimization for backward propagating signals aimed at maximizing the probe output OSNR, while keeping the minimum Brillouin pump-probe power difference within the fiber larger than $17.5 \mathrm{~dB}$ (allowing some power margin) to avoid nonlocal effects. Fig. 1 reports the OSNR (solid lines) and minimum pump-probe difference (dotted lines) versus backward Raman pump power for several input probe power levels. Following a procedure similar to what described in [6], the optimum power levels have been found to be: 1) $24.6 \mathrm{dBm}$ for the backward Raman pump, 2) $25.8 \mathrm{dBm}$ for the forward Raman pump, 3) $-20 \mathrm{dBm}$ for the probe signal, and 4) $10 \mathrm{dBm}$ for the Brillouin pump.

In addition, based on numerical simulations carried out on the probe-signal RIN-transfer crosstalk, as described in [6], a low-RIN backward Raman pump is required in order to avoid a significant RIN-transfer induced penalty to the probe, and to achieve an acceptable BOTDA performance at $120 \mathrm{~km} \mathrm{[5],} \mathrm{[6].}$

On the other hand, to allow for linear Brillouin gain and to avoid trace distortions due to pattern-dependent acoustic-wave

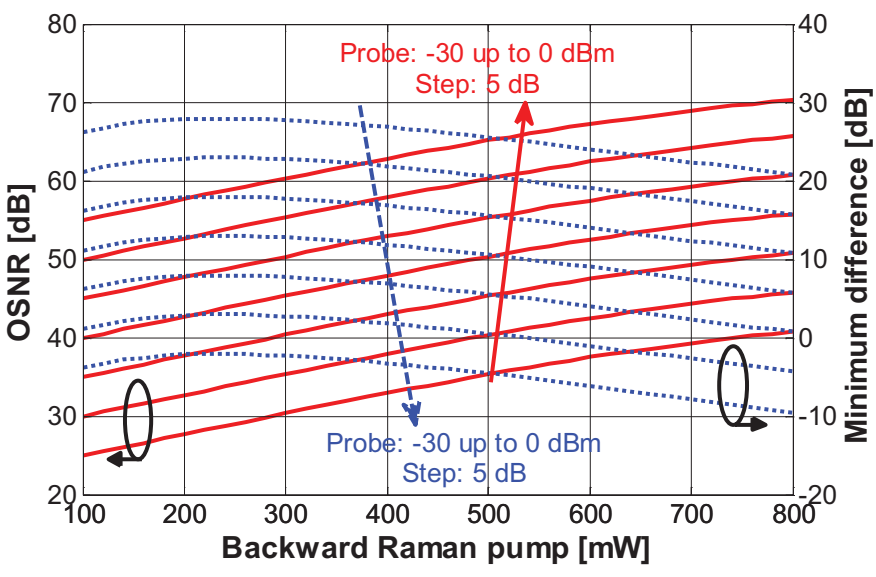

Fig. 1. Probe OSNR at the receiver (solid lines, left axis) and minimum Brillouin pump-probe difference (dotted lines, right axis) versus Raman pump power, for probe power levels ranging from -30 to $0 \mathrm{dBm}$.

pre-excitation [9] or the above-mentioned code-dependent Raman amplification, our system requires the use of RZ coded pulses with optimized duty cycle. The transient behavior of the acoustic wave and the respective SBS gain in the probe signal have been analyzed for our scheme by solving the three-wave SBS transient model, as described in [9]. Numerical results point out that a bit slot equal to $80 \mathrm{~ns}$ can be used in this case (12.5\% duty cycle), allowing for a significant acoustic wave damping (during $70 \mathrm{~ns}$ ) and an adequate SBS gain recovery.

\section{EXPERIMENTAL RESULTS}

The setup used in the experiments is shown in Fig. 2. The CW beam from a distributed-feedback (DFB) laser at $1550 \mathrm{~nm}$ is split using a 3-dB optical coupler. In the Brillouinpump branch, an Erbium-doped fiber amplifier (EDFA) and a Mach-Zehnder modulator (MZM), which is controlled by a waveform generator (WFG), have been used to generate highpower RZ Simplex-coded pulse sequences with 127 bit.

In the second branch, a double-sideband probe signal is generated by intensity-modulating the $\mathrm{CW}$ light with an MZM driven by a microwave generator. A variable optical attenuator (VOA) is used to adjust the input probe power, while a passive polarization scrambler (PS) for CW signals, based on a fiberbased unbalanced Mach-Zehnder interferometer (see details in [10]), is used to reduce polarization-dependent gain (PDG).

Bidirectional DRA is implemented coupling two Raman pumps at $1450 \mathrm{~nm}$ into a $120 \mathrm{~km}$ single-mode fiber (SMF). To reduce Raman PDG a depolarized fiber Raman laser (FRL) is used as forward Raman pump, while the backward pump is implemented in a low-RIN scheme $(<-130 \mathrm{~dB} / \mathrm{Hz})$ using two Fabry-Perot (FP) lasers multiplexed in orthogonal polarization.

At the receiver, the coded probe signal is coupled into a linear-gain EDFA (used as a preamplifier) employing a 3-port optical circulator. Another circulator and a fiber Bragg grating (FBG, 6-GHz) have been used to filter out the ASE noise of the preamplifier, the residual suppressed carrier, the Brillouin anti-Stokes line and the Rayleigh signal. Finally, a $125-\mathrm{MHz}$ 


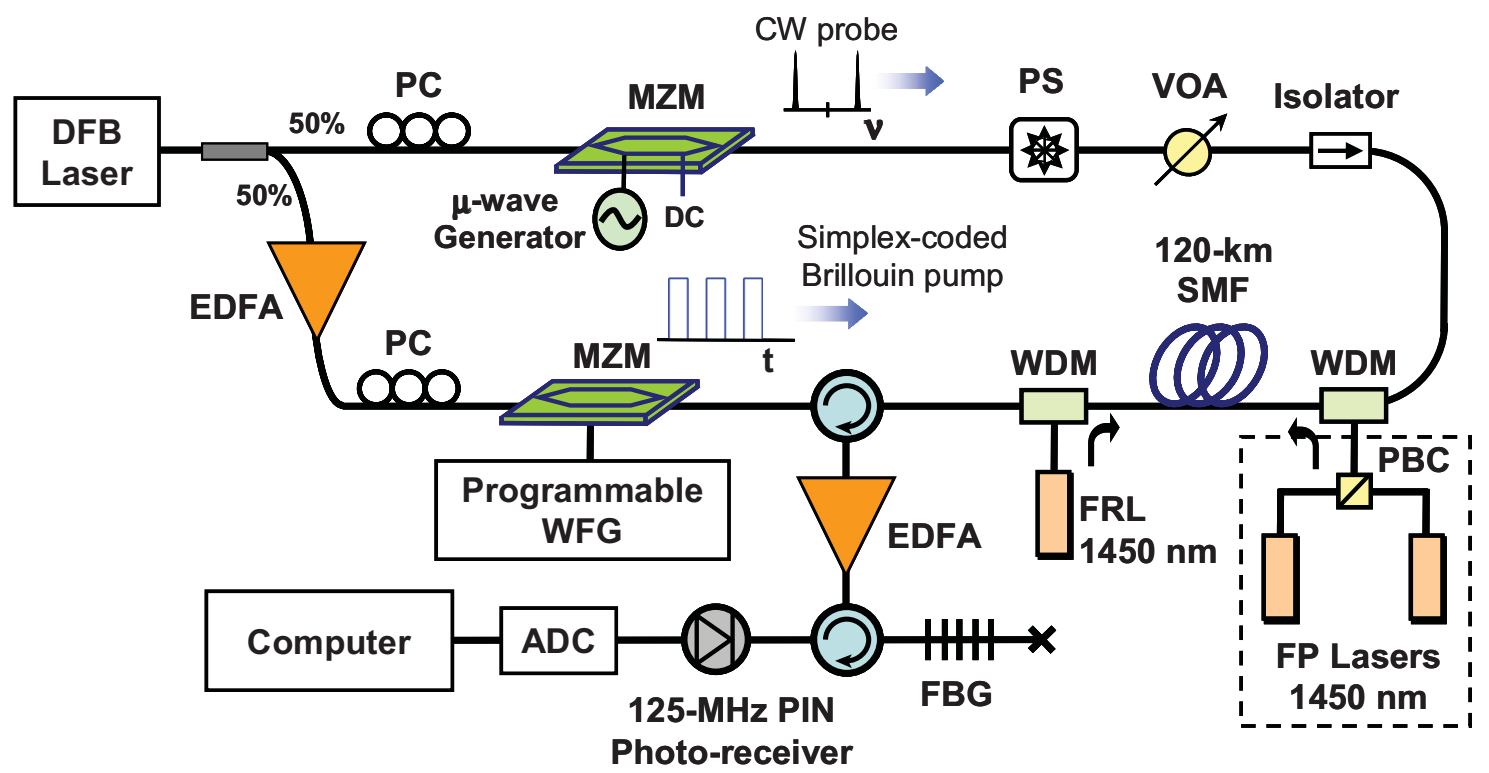

Fig. 2. Experimental setup.

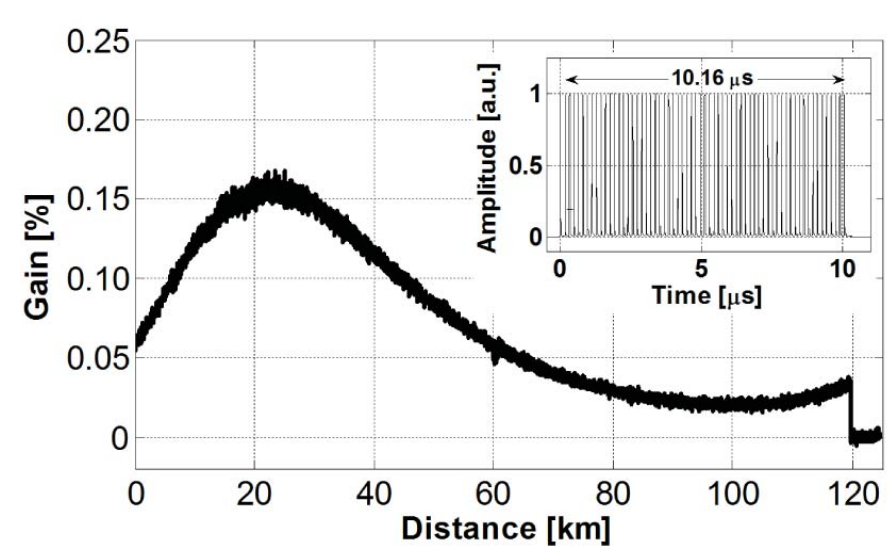

Fig. 3. Decoded BOTDA trace at the maximum Brillouin gain. Inset: detected stream of a codeword for the Brillouin pump after $120-\mathrm{km}$ propagation.

PIN photo-receiver and an analog-to-digital converter (ADC) have been connected to a computer for trace acquisition.

Coded BOTDA traces have been measured with a total of $8 \mathrm{k}$ time-averaged traces per frequency. In Fig. 3 we can observe a decoded BOTDA trace (at the peak BGS frequency) with an enhanced SNR, resulting from the optimized system.

Note that the use of optimized RZ pulses (allowing linear SBS amplification [9]) and a linear-gain preamplifier avoids nonlinear distortion of the coded traces; hence, standard linear decoding process can be used. The inset in Fig. 3 reports an undistorted residual coded Brillouin pump for a specific codeword (after propagation and amplification along $120 \mathrm{~km}$ ), confirming that the DRA does not distort codewords, as happens with other optical amplifiers (e.g. EDFAs).

The decoded BGS versus distance is reported in Fig. 4. We verified that the full-width at half-maximum (FWHM) of the BGS keeps almost constant throughout the sensing fiber, resulting in $\sim 100 \mathrm{MHz}$ as expected for the 10ns-pulsewidth

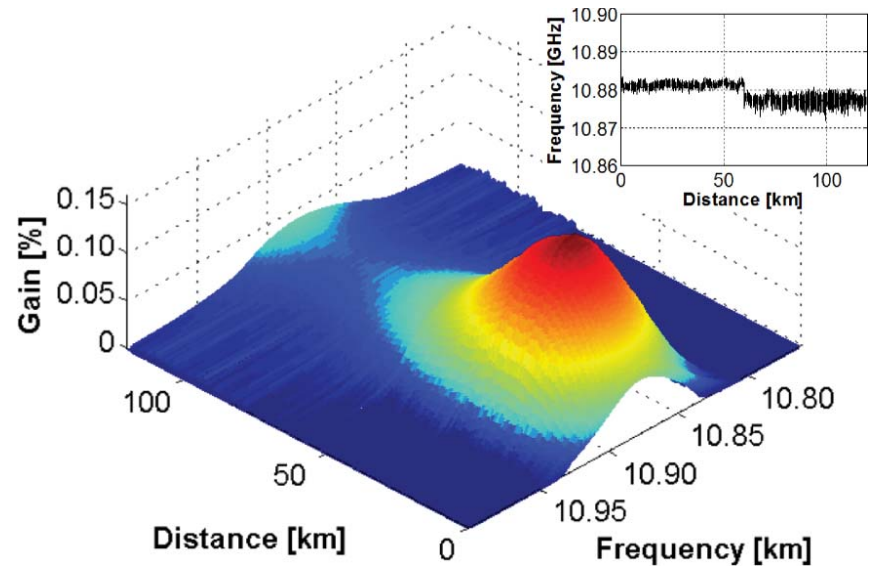

Fig. 4. Brillouin gain spectrum. Inset: BFS parameter along the fiber.

case [9]. This indicates that, thanks to the RZ-coding with optimized duty cycle, no distortion of the BGS (induced by phonon pre-excitation) is occurring; also nonlinear effects have been avoided in the above-mentioned power-optimization process. The BFS has then been estimated as a function of the distance from the decoded BGS, as shown in the inset of Fig. 4. Since the sensing fiber is composed of two different $60 \mathrm{~km}$-long fiber spools, we can notice a slight frequency difference ( $\sim 5 \mathrm{MHz}$, related to manufacturing) in the BFS for the two different spools (both at room temperature of $27.5^{\circ} \mathrm{C}$ ). Considering that such a frequency difference is small in comparison to the BGS linewidth $(\sim 100 \mathrm{MHz})$, a continuous SBS interaction is then expected to take place along the whole fiber length, corresponding to the worst-case condition in terms of pump depletion effects. Even in such a case, due to the use of a double-sideband probe and optimized DRA, the level of pump depletion was kept as low as $0.8 \%$ (measured as the residual Brillouin pump variation with and without SBS interaction), thus avoiding potential measurement errors. 


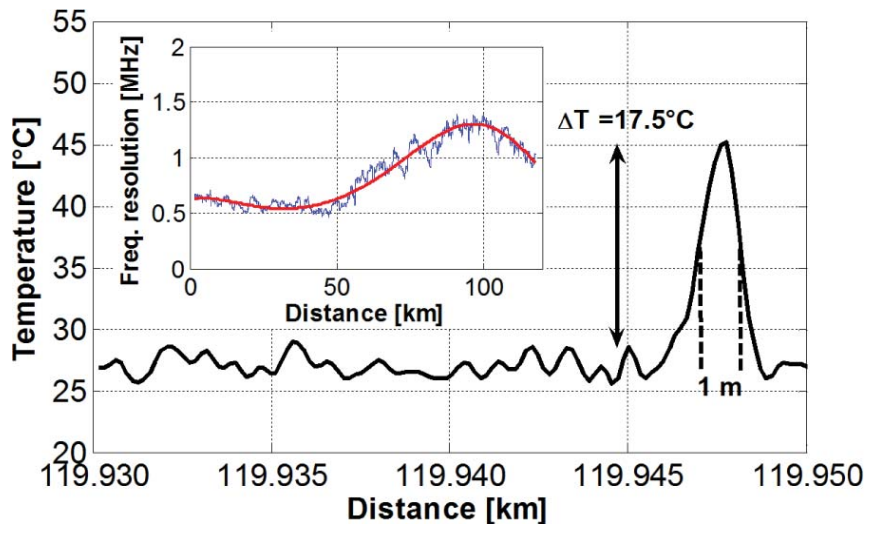

Fig. 5. Demonstration of $1-\mathrm{m}$ hot-spot detection, at $\sim 120-\mathrm{km}$ distance. Inset: measurand resolution (in frequency) versus distance.

To verify the real spatial-resolution capabilities of the implemented sensor, one meter of fiber at $\sim 120 \mathrm{~km}$ distance has been placed inside a temperature-controlled chamber (TCC) at $45{ }^{\circ} \mathrm{C}$. Fig. 5 shows the measured temperature profile only for the last $20 \mathrm{~m}$ of fiber, where we can clearly observe a 1-meter hot-spot of $17.5^{\circ} \mathrm{C}$. This result corresponds to the first demonstration of a BOTDA sensor with capability to measure temperature and strain with a spatial resolution of $1.0 \mathrm{~m}$ along a $120 \mathrm{~km}$ range, and attaining at the same time a significantly enhanced temperature-strain resolution with respect to previously reported long-range BOTDA sensors [4]-[7]. The measurand resolution is evaluated as the standard deviation of the BFS as a function of the distance, as shown in the inset of Fig. 5. We can see that the worst resolution is attained at $\sim 100 \mathrm{~km}$ distance (where the lowest SNR is observed), resulting in a worst-case resolution of $1.3 \mathrm{MHz}$, corresponding to temperature and strain resolutions of $1.3^{\circ} \mathrm{C}$ and $26 \mu \varepsilon$.

\section{CONCLUSiON}

In conclusion, we reported on the input parameters optimization of a BOTDA sensor employing distributed lowRIN bidirectional Raman amplification combined with the use of Simplex codes with an optimal RZ modulation format, allowing for strain-temperature sensing with $1 \mathrm{~m}$ spatial resolution along a $120 \mathrm{~km}$-long single-mode fiber.

\section{REFERENCES}

[1] A. Minardo, R. Bernini, L. Zeni, L. Thévenaz, and F. Briffod, "A reconstruction technique for long-range stimulated Brillouin scattering distributed fibre-optic sensors: Experimental results," Meas. Sci. Technol., vol. 16, no. 4, pp. 900-908, 2005.

[2] L. Thévenaz, S. F. Mafang, and J. Lin, "Impact of pump depletion on the determination of the Brillouin gain frequency in distributed fiber sensors," Proc. SPIE, vol. 7753, pp. 775322-1-775322-4, May 2011.

[3] D. Lee, H. Yoon, P. Kim, J. Park, N. Kim, and N. Park, "SNR enhancement of OTDR using biorthogonal codes and generalized inverses," IEEE Photon. Technol. Lett., vol. 17, no. 1, pp. 163-165, Jan. 2005.

[4] M. A. Soto, G. Bolognini, and F. D. Pasquale, "Long-range simplexcoded BOTDA sensor over $120 \mathrm{~km}$ distance employing optical preamplification," Opt. Lett., vol. 36, no. 2, pp. 232-234, 2011.

[5] X. Angulo-Vinuesa, et al., "Raman-assisted Brillouin distributed temperature sensor over $100 \mathrm{~km}$ featuring $2 \mathrm{~m}$ resolution and $1.2{ }^{\circ} \mathrm{C}$ uncertainty," J. Lightw. Technol., vol. 30, no. 8, pp. 1060-1065, Apr. $15,2012$.

[6] M. A. Soto, G. Bolognini, and F. Di Pasquale, "Optimization of long-range BOTDA sensors with high resolution using first-order bidirectional Raman amplification," Opt. Express, vol. 19, no. 5, pp. 44444457, 2011.

[7] X.-H. Jia, et al., "Experimental demonstration on 2.5-m spatial resolution and $1{ }^{\circ} \mathrm{C}$ temperature uncertainty over long-distance BOTDA with combined Raman amplification and optical pulse coding," IEEE Photon. Technol. Lett., vol. 23, no. 7, pp. 435-437, Apr. 1, 2011.

[8] M. N. Islam, Raman Amplifiers for Telecommunications. New York: Springer-Verlag, 2004.

[9] M. A. Soto, G. Bolognini, and F. Di Pasquale, "Analysis of pulse modulation format in coded BOTDA sensors," Opt. Express, vol. 18, no. 17, pp. 14878-14892, 2010.

[10] S. Diaz, S. F. Mafang, M. Lopez-Amo, and L. Thévenaz, "A highperformance optical time-domain brillouin distributed fiber sensor," IEEE Sensors J., vol. 8, no. 7, pp. 1268-1272, Jul. 2008. 